\title{
A Rare Case Presenting With Acute Stroke-Like Clinic: Todd's Paralysis in the Setting of Frontal Dural-Based Tumor
}

\author{
Halil Ondera, b
}

\begin{abstract}
Herein, I present an 86-year-old male patient presenting with strokelike symptoms in which diffusion-restricted lesion in diffusion weighted imaging (DWI) was firstly evaluated as ischemic stroke. However, the lesion was found to be atypical for ischemic stroke after meticulous evaluation of the DWI. Detailed neuroimaging studies and re-evaluation of the clinic led to the proper diagnosis of Todd's paralysis in the setting of dural-based tumor. Via the presentation of this case and literature data, I draw attention to the importance of correct interpretation of DWI for proper diagnosis. I also remark the importance of multimodal evaluation of the patients before administering specific, but potentially harmful treatments such as thrombolytic drugs.
\end{abstract}

Keywords: Ischemic stroke; Stroke mimics; Meningioma; DWI; Todd's paralysis

\section{Introduction}

Diagnostic safety in ischemic stroke has increasingly been emphasized for optimum clinical outcome of the patients. At this point, "stroke mimics" (false-positive cases in other words) constitute a crucial diagnostic challenge in emergency department which may cause unnecessary and potentially harmful treatment options [1]. Herein, I present an interesting patient presenting with a stroke-like clinic who eventually received the final diagnosis of Todd's paralysis in the setting of left frontal dural-based tumor. Via the presentation of this case, I will emphasize the importance of awareness of "stroke mimics" in emergency practice and suggest some critical points for the proper diagnosis.

\section{Case Report}

A-86-year-old male with a medical history of mild-degree dementia and ischemic cerebrovascular disease (9 years ago,

Manuscript submitted September 20, 2018, accepted October 26, 2018

aNeurology Clinic, Yozgat City Hospital, Yozgat, Turkey

${ }^{b}$ Corresponding Author: Halil Onder, Yozgat City Hospital, Neurology Clinic,

Yozgat, Turkey. Email: halilnder@yahoo.com

doi: https://doi.org/10.14740/jnr498 characterized by dysarthria due to ischemia of right corona radiata) was admitted due to right-sided paralysis and confusion which was realized $2 \mathrm{~h}$ prior to admission. Of note, no history of previous seizure, head trauma or brain surgery was taken. On neurological examination, he was non-cooperative and non-orientated. Motor examination revealed paralysis on the right side, predominant in the lower extremity ( $3 / 5$ on Medical Research Council (MRC)). Diffusion weighted imaging (DWI), performed $3 \mathrm{~h}$ after clinical onset, showed diffusionrestricted region in the left frontal area (Fig. 1). First evaluation in the emergency department led to a diagnosis of left frontal ischemic stroke and promptly neurology consultation was demanded. However, after re-evaluation of DWI, lesion site was found to be atypical for ischemic stroke which was incompatible with a vascular territory, demonstrating a frontal convexity and clearly boundaries. At this point, a re-interrogation of the history was conducted which revealed that a short-term convulsive attack in his right side was observed at onset of clinical manifestations. Cranial enhanced MRI was performed which showed contrast enhancement and extra-axial T2 isointense frontal lesion, compatible with frontal duralbased tumor (Fig. 2). Routine EEG showed sharp wave paroxysms in the left frontotemporal lobe. Taken together, Todd's paralysis in the setting of left frontal meningioma (possibly) was diagnosed. In accordance with this, the patient recovered totally in the following $12 \mathrm{~h}$ after admission. Tumor surgery was suggested; however, the patients' relatives rejected surgery; hence, the patient was discharged on therapy of $300 \mathrm{mg}$ $(3 \times 100 \mathrm{mg})$ phenytoin, with a follow-up plan in outpatient clinics. Anti-aggregant therapy was also continued due to previous ischemic stroke.

\section{Discussion}

Currently, ischemic stroke is one of the leading causes of longterm disability and death in the USA [2]. The development of recent therapies including thrombolysis intervention and invasive methods such as mechanical thrombectomy, angioplasty and stenting, which are applicable within the therapeutic window, has improved the survival rates and patients' quality of life. Therefore, early and accurate diagnosis has been emphasized as the basic point for achieving improved outcome. However, a significant number of patients with stroke-like symptoms do not have stroke, also termed "stroke mimics", and recent studies showed that $3-13 \%$ of patients were mistakenly diagnosed and treated as acute stroke $[2,3]$. The most 


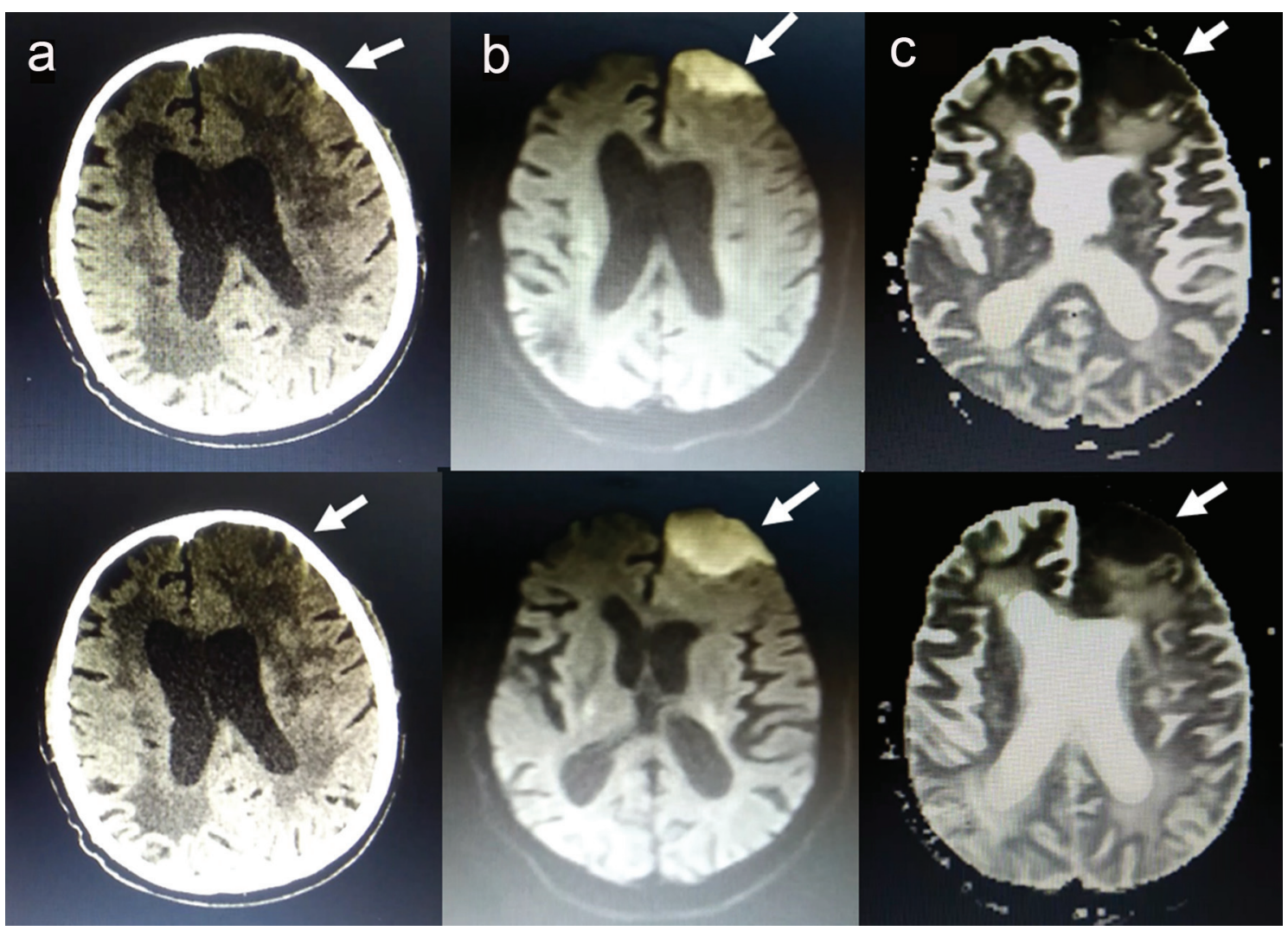

Figure 1. (a) Computed tomography showing hypodense lesion in the left frontal area. (b) High signal in the left frontal area on DWI. (c) Low signal on apparent diffusion coefficient (ADC).

common stroke mimic diagnoses include migraine, epileptic seizures, psychogenic or conversion disorder, toxic-metabolic abnormalities and intracranial tumors [4, 5]. Remarkably, misdiagnoses of these entities as stroke can lead to unnecessary and potentially harmful interventions, such as thrombolysis intervention. In the light of these data, an intensive effort has been conducted to increase awareness of "stroke mimics" as well as proper diagnosis of these entities $[1,6]$. Therefore, some authors suggested performing a "stroke mimic scale" to distinguish these entities. Remarkably, neuroimaging studies constitute a critical and essential point for proper diagnosis. DWI was firstly introduced in the 1990s and quickly adopted in the evaluation of acute stroke [7]. In ischemic stroke, diffusion restriction can be observed within minutes to less than $1 \mathrm{~h}$ from onset of the clinic and it is highly sensitive in the diagnosis of ischemic injury for which it has been a vital paraclinical tool in evaluation of patients with stroke [7]. However, the utility of DWI can differ according to the interpreter's knowledge and experience. Furthermore, diagnosis of stroke is a multimodal work requiring interpretation of both clinical clues and neuroimaging findings.

In this report, I present a rare patient presenting with stroke-like symptoms in which diffusion-restricted lesion in DWI was firstly evaluated as ischemic stroke in the emergency practice. However, the lesion was found to be atypical for ischemic stroke after meticulous evaluation of the DWI. Hence, contrast-enhanced MRI was performed, showing ho- mogeneous contrast enhancement, hypointense on T2-weighted images and isointense to gray matter on T2-weighted images, which were compatible with dural-based tumor. In the following course, the symptoms of right-sided paralysis and confusion recovered and routine EEG showed epileptiform discharges supporting the diagnosis of Todd's paralysis. A limitation of this report may be that transient ischemic attack cannot be totally ruled out in this patient especially considering the previous ischemic cerebrovascular disease. Of note, we also planned to perform the routine work-up for previous ischemic stroke in the follow-up polyclinic visits. Via the presentation of this interesting case and literature data emphasizing many other causes of diffusion restriction on DWI, I draw attention to the importance of correct interpretation of DWI for accurate diagnosis of stroke. Furthermore, this case may also remark the importance of multimodal evaluation for the patients before administering specific, but potentially harmful treatments such as thrombolytic drugs. In my opinion, illustration of these rare samples may aid to increase the awareness of stroke mimics and therefore, prevent unnecessary interventions such as thrombolytic intervention and develop clinical outcomes.

\section{Conflict of Interest}

None. 


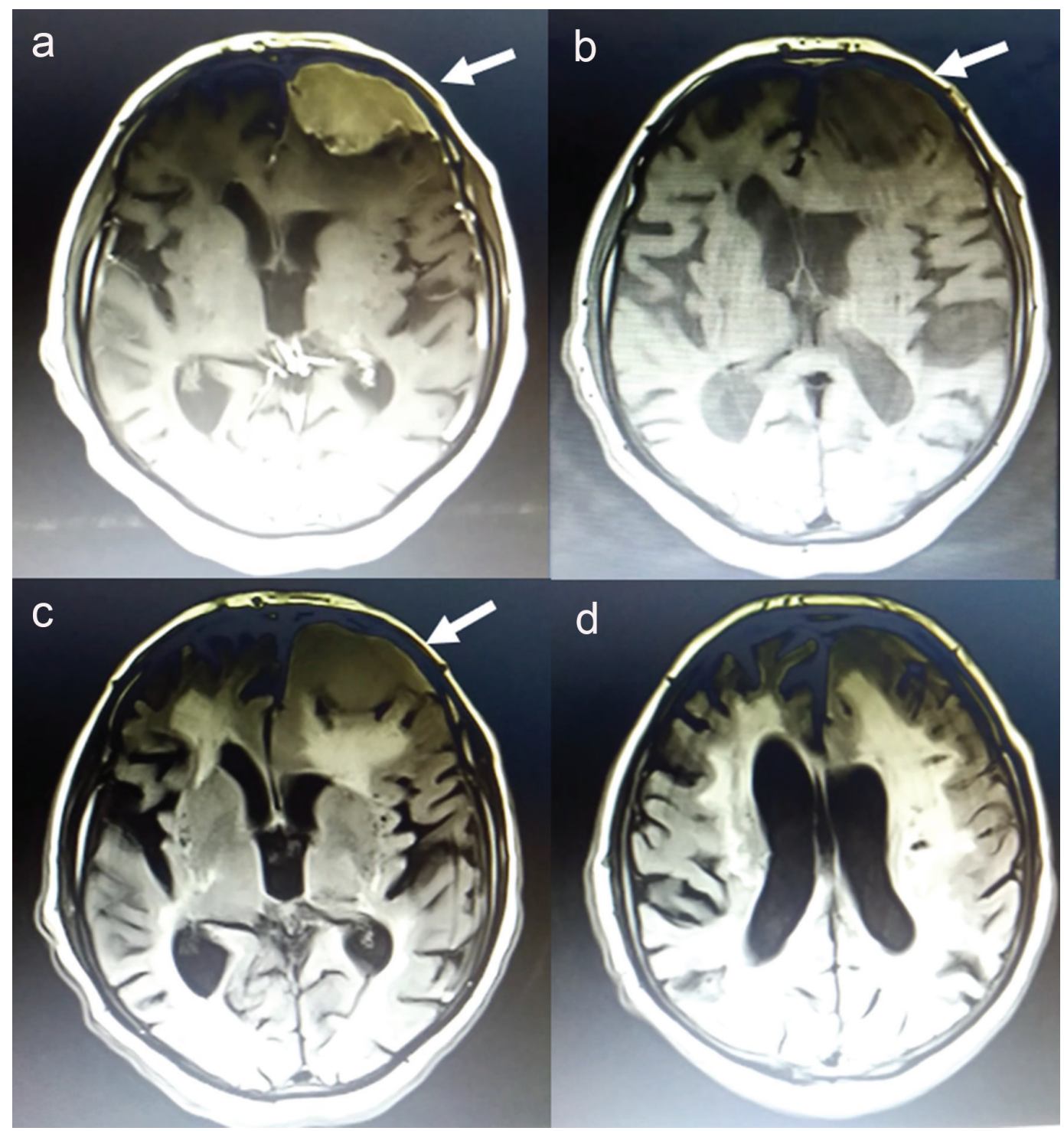

Figure 2. Cranial enhanced MRI was performed which showed homogenous contrast enhancement (a), T1 hypointense (b) and extra-axial T2 isointense (c) frontal lesion, compatible with frontal dural-based tumor. Fluid-attenuated inversion recovery (FLAiR) image showing chronic, subcortical lesions which are ischemic in nature (d).

\section{Funding}

None.

\section{References}

1. Liberman AL, Prabhakaran S. Stroke chameleons and stroke mimics in the emergency department. Curr Neurol Neurosci Rep. 2017;17(2):15.

2. Libman RB, Wirkowski E, Alvir J, Rao TH. Conditions that mimic stroke in the emergency department. Implications for acute stroke trials. Arch Neurol. 1995;52(11):1119-1122.

3. Hand PJ, Kwan J, Lindley RI, Dennis MS, Wardlaw JM.
Distinguishing between stroke and mimic at the bedside: the brain attack study. Stroke. 2006;37(3):769-775.

4. Liu X, Almast J, Ekholm S. Lesions masquerading as acute stroke. J Magn Reson Imaging. 2013;37(1):15-34.

5. Zinkstok SM, Engelter ST, Gensicke H, Lyrer PA, Ringleb PA, Artto V, Putaala J, et al. Safety of thrombolysis in stroke mimics: results from a multicenter cohort study. Stroke. 2013;44(4):1080-1084.

6. Khan NI, Chaku S, Goehl C, Endris L, Mueller-Luckey G, Siddiqui FM. Novel Algorithm to Help Identify Stroke Mimics. J Stroke Cerebrovasc Dis. 2018;27(3):703-708.

7. Allen LM, Hasso AN, Handwerker J, Farid H. Sequencespecific MR imaging findings that are useful in dating ischemic stroke. Radiographics. 2012;32(5):1285-1297; discussion 1297-1289. 